\title{
The Cordial Labeling for the Four-Leaved Rose Graph
}

\author{
Ashraf Elrokh \\ Department of Math, Faculty of Science, Menoufia University, Shebeen Elkom, Egypt \\ Email address: \\ el-rokh@excite.com \\ To cite this article: \\ Ashraf Elrokh. The Cordial Labeling for the Four-Leaved rose graph. Applied and Computational Mathematics. \\ Vol. 7, No. 4, 2018, pp. 203-211. doi: 10.11648/j.acm.20180704.14
}

Received: June 22, 2018; Accepted: August 31, 2018; Published: October 15, 2018

\begin{abstract}
A cactus graph with four blocks which are all cycles, not necessarily be of the same size, is called four-leaved rose graph and denoted by $L_{n, m, k, s}$, where $\mathrm{n}, \mathrm{m}, \mathrm{k}$ and $\mathrm{s}$ represent she sizes of the four cycles. A cordial graph is a graph whose vertices and edges have 0-1 labeling in such a way that the number of vertices (edges) labelled with zeros and the number of vertices (edges) labelled with ones differ absolutely by at most one .In this paper, we study this graph in detail and show that any four-leaved rose graph is cordial for all $n, m, k$ and $s$ except possibly at $n, m$ are odd with $(k+s)=0(\bmod 4)$ or $n, m$ are even with $(k+s)=2(\bmod 4)$. Our technique depends on the methods that partition off the set of positive integers and then use suitable labeling in each division of the partition to achieve our results. AMS classification $05 \mathrm{C} 76,05 \mathrm{C} 78$
\end{abstract}

Keywords: Cactus Graph, Cordial Labeling, Four-Leaved Rose Graph

\section{Introduction}

A graph coloring is one of the oldest problems of graph theory. The origins of graph labeling may be back to 1852 when De Morgen asked : what is the least possible number of colors needed to fill in any map on the plane ?. He is observed that only four colors were necessary in order to ensure that adjacent countries were give different colors. The concept of graph labeling was introduced during the sixties of the last century by Rosa [1] or Graham and Sloane [2]. There are many contributions and different kinds of labeling [3-6]. An excellent reference on this subject is the survey by Gallian [7]. Suppose that $G=(V, E)$ is a graph, where $V$ is the set of its vertices and $E$ is the set of its edges. Throughout, it is assumed $G$ is connected, finite, simple and undirected. A binary vertex labeling of $G$ is a mapping $f: V \rightarrow\{0,1\}$ in which $f(u)$ is said to be the labeling of $u \in V$. For an edge $e=u v \in E$, where $u, v \in V$, the introduced edge labeling $f^{*}: E \rightarrow\{0,1\}$ is defined by the formula $f^{*}(u v)=(f(u)+$ $f(v))(\bmod 2)$. Thus, for any edge $e, f^{*}(e)=0$ if its two vertices have the same labeling and $f^{*}(e)=1$ if they have different labeling. Let us denote $v_{0}$ and $v_{1}$ be the numbers of vertices labeled by 0 and 1 in $V$ respectively, and let $e_{0}$ and $e_{1}$ be the corresponding numbers of edge in $E$ labeled by 0 and 1 respectively. A binary vertex labeling $f$ of $G$ is said to be cordial if $\left|v_{0}-v_{1}\right| \leq 1$ and $\left|e_{0}-e_{1}\right| \leq 1$ hold. A graph $G$ is cordial if it has a cordial labeling. The original concept of cordial graphs is due to Chait [8].

A cactus is a graph in which any two cycles have most one vertex in common. The cycles may or may not be of the same sizes. The four-leaved rose graph, denoted by $L_{n, m, k, s}$, where $n, m, k, s$ are the sizes of four cycles.

In [9], Kirchherr discussed the cordiality of a cactus whose blocks are cycles of the same sizes. Similar result is given by $\mathrm{S}$. M. Lee and A. Liu [10]. In this paper, we consider the cactus with different sizes. We show that the four-leaved rose graph is cordial for all $n, m, k$ and $s$ except if $(n, m$ are odd and $(k+s) \equiv$ $0(\bmod 4))$ or $(n, m$ are even and $(k+s) \equiv 2(\bmod 4))$.

\section{Terminologies and Notations}

A cycle with $n$ vertices and $n$ edges is denoted by $C_{n}$. Given one cycle of the four-leaved rose graph with $4 r$ vertices, we let $L_{4 r}$ denote the labeling $00110011 \ldots 0011$ (repeated $r$-times), $\bar{L}_{4 r}$ denote the labeling 1100 $1100 \ldots 1100$ (repeated $r$-times), $S_{4 r}$ denotes the labeling $10011001 \ldots 1001$ (repeated $r$-times) and $\bar{S}_{4 r}$ denotes the labeling $01100110 \ldots 0110$ (repeated r-times). In most cases, we then modify this by adding symbols at one end or the other (or both); Similarly $L_{4 r} 101$ denotes the labeling 0011 $0011 \ldots 0011101$ for one cycle of the four-leaved rose graph $L_{4 r+3,4 x+3,4 t+3,4 b+3}$ when $r \geq 1$ and 101 when $r=0$, 
$1 \bar{L}_{4 r}$ is the labeling $111001100 \ldots 1100$ for one cycle of the four-leaved rose graph $L_{4 r+2,4 x+2,4 t+2,4 b+2}$ when $r \geq 1$ and 1 when $r=0$ and $0 \bar{L}_{4 r} 1$ is the labeling $0 \quad 1100$ $1100 \ldots 11001$ for one cycle of the four-leaved rose graph $L_{4 r+3,4 x+3,4 t+3,4 b+3}$ when $r \geq 1$ and 01 when $r=0$, and so on.

For a given labeling of the four leaved rose graph, we denote $v_{i}$ and $e_{i}$ to represent the number of vertices and edges of the graph, respectively, labeled by i. It is easy to verify that $v_{0}-v_{1}=\left(x_{0}-x_{1}\right)+\left(y_{0}-y_{1}\right)+\left(z_{0}-z_{1}\right)+$ $\left(h_{0}-h_{1}\right)$ and $e_{0}-e_{1}=\left(a_{0}-a_{1}\right)+\left(b_{0}-b_{1}\right)+\left(c_{0}-\right.$ $\left.c_{1}\right)+\left(d_{0}-d_{1}\right)$. It is easy to see that $L_{n, m, k, s}$ is isomorphic $L_{n, m, s, k}, L_{m, n, k, s}, L_{m, n, s, k}, L_{n, k, m, s}, L_{s, k, m, n}, L_{s, m, k, n}, L_{n, s, k, m}$, $L_{k, s, n, m}$ and $L_{k, m, n, s}$.

\section{The Four-Leaved Rose Graph}

In this section, we show that the four-leaved rose graph is cordial except if $(n, m$ are odd and $(k+s) \equiv 0(\bmod 4))$ or $(n, m$ are even and $(k+s) \equiv 2(\bmod 4))$. This target will be achieved as a consequence of the following series of lemmas.

Lemma 3.1. The four-leaved rose graph is cordial if $m, n=3$ and $k, s$ are odd.

Proof. We choose the labeling [110:00:11:10] for $L_{3,3,3,3}$. Therefore $x_{0}=1, x_{1}=2, a_{0}=1, a_{1}=2, y_{0}=2$, $y_{1}=0, b_{0}=3, b_{1}=0, z_{0}=0, z_{1}=2, c_{0}=1, c_{1}=2$, $h_{0}=1, h_{1}=1, d_{0}=1$ and $d_{1}=2$. Hence $v_{0}-v_{1}=$ $\left(x_{0}-x_{1}\right)+\left(y_{0}-y_{1}\right)+\left(z_{0}-z_{1}\right)+\left(h_{0}-h_{1}\right)=-1 \quad$ and $e_{0}-e_{1}=\left(a_{0}-a_{1}\right)+\left(b_{0}-b_{1}\right)+\left(c_{0}-c_{1}\right)+\left(d_{0}-d_{1}\right)=$ 0 . Thus $L_{3,3,3,3}$ is cordial. Now, we need to study the following cases for $k, s>4$.

Case (1.1): $k, s \equiv 1(\bmod 4)$.

Suppose that $k=4 t+1, s=4 b+1$, where $t, b \geq 1$. We choose the labeling [110:10: $\bar{L}_{4 t}: \bar{L}_{4 b}$ ] for $L_{3,3,4 t+1,4 b+1}$. Therefore $x_{0}=0, x_{1}=2, a_{0}=1, a_{1}=2, y_{0}=1, y_{1}=1$, $b_{0}=1, b_{1}=2, z_{0}=2 t, z_{1}=2 t, c_{0}=2 t+1, c_{1}=2 t$, $h_{0}=2 b, h_{1}=2 b, d_{0}=2 b+1$ and $d_{1}=2 b$. Hence $v_{0}-v_{1}=\left(x_{0}-x_{1}\right)+\left(y_{0}-y_{1}\right)+\left(z_{0}-z_{1}\right)+\left(h_{0}-\right.$ $\left.h_{1}\right)=-1$ and $e_{0}-e_{1}=\left(a_{0}-a_{1}\right)+\left(b_{0}-b_{1}\right)+\left(c_{0}-\right.$ $\left.c_{1}\right)+\left(d_{0}-d_{1}\right)=0$. Thus $L_{3,3,4 t+1,4 b+1}$ is cordial.

Case $(2.1): k, s \equiv 3(\bmod 4)$.

Suppose that $k=4 t+3, s=4 b+3$, where $t, b \geq 1$. We choose the labeling [011:10: $\left.\bar{L}_{4 t} 01: 1 L_{4 b} 0\right]$ for $L_{3,3,4 t+3,4 b+3}$. Therefore $x_{0}=1, x_{1}=2, a_{0}=1, a_{1}=2$, $y_{0}=1, y_{1}=1, b_{0}=1, b_{1}=2, z_{0}=2 t+1, z_{1}=2 t+1$, $c_{0}=2 t+3, c_{1}=2 t, h_{0}=2 b+1, h_{1}=2 b+1, d_{0}=$ $2 b+1$ and $d_{1}=2 b+2$. Hence $v_{0}-v_{1}=\left(x_{0}-x_{1}\right)+$ $\left(y_{0}-y_{1}\right)+\left(z_{0}-z_{1}\right)+\left(h_{0}-h_{1}\right)=-1$ and $e_{0}-e_{1}=$ $\left(a_{0}-a_{1}\right)+\left(b_{0}-b_{1}\right)+\left(c_{0}-c_{1}\right)+\left(d_{0}-d_{1}\right)=0$. Thus $L_{3,3,4 t+3,4 b+3}$ is cordial.

Lemma 3.2. The four-leaved rose graph is not cordial if $m, n=3$ and $k, s$ are even.

Proof. It's easy to verify that the degree of all vertices of $L_{n, m, k, s}$ is even, and $L_{n, m, k, s}$ is an Eulerian graph with size is congruent to $2(\bmod 4)$, then from Cahit's theorem [7], $L_{n, m, k, s}$ is not cordial.

Lemma 3.3. The four-leaved rose graph is cordial if $m, n \equiv 0(\bmod 4)$ and $k, s$ are even.

Proof. $L_{4,4,3,3}$ is not cordial since it's isomorphic to $L_{3,3,4,4}$. Now, we need to study the following cases for $k, s \geq 4$.

Case (1.3): $k, s \equiv 0(\bmod 4)$.

Suppose that $k=4 t, s=4 b$, where $t, b \geq 0$. We choose the labeling $\left[L_{4 r}: L_{4 x} 100: 101 L_{4 t}: 110 \bar{L}_{4 b}\right]$ for $L_{4 r, 4 x, 4 t, 4 b}$. Therefore $x_{0}=2 r, x_{1}=2 r, a_{0}=2 r, a_{1}=2 r, y_{0}=2 x+$ $2, y_{1}=2 x+1, b_{0}=2 x+2, b_{1}=2 x+2, z_{0}=2 t+1$, $z_{1}=2 t+2, \quad c_{0}=2 t+2, \quad c_{1}=2 t+2, \quad h_{0}=2 b+1$, $h_{1}=2 b+2, d_{0}=2 b+2$ and $d_{1}=2 b+2$. Hence $v_{0}-v_{1}=\left(x_{0}-x_{1}\right)+\left(y_{0}-y_{1}\right)+\left(z_{0}-z_{1}\right)+\left(h_{0}-\right.$

$\left.h_{1}\right)=-1$ and $e_{0}-e_{1}=\left(a_{0}-a_{1}\right)+\left(b_{0}-b_{1}\right)+\left(c_{0}-\right.$ $\left.c_{1}\right)+\left(d_{0}-d_{1}\right)=0$. Thus $L_{4 r, 4 x, 4 t, 4 b}$ is cordial.

Case $(2.3): k, s \equiv 2(\bmod 4)$.

Suppose that $k=4 t+2, s=4 b+2$, where $t, b \geq 1$. We choose the labeling $\left[L_{4 r}: L_{4 x} 100: \bar{L}_{4 t} 1: \bar{S}_{4 b} 1\right]$ for $L_{4 r, 4 x, 4 t+2,4 b+2}$. Therefore $x_{0}=2 r, x_{1}=2 r, a_{0}=2 r$, $a_{1}=2 r, y_{0}=2 x+2, y_{1}=2 x+1, b_{0}=2 x+2, b_{1}=$ $2 x+2, z_{0}=2 t, \quad z_{1}=2 t+1, \quad c_{0}=2 t+2, \quad c_{1}=2 t$, $h_{0}=2 b, h_{1}=2 b+1, d_{0}=2 b$ and $d_{1}=2 b+2$. Hence $v_{0}-v_{1}=\left(x_{0}-x_{1}\right)+\left(y_{0}-y_{1}\right)+\left(z_{0}-z_{1}\right)+\left(h_{0}-\right.$ $\left.h_{1}\right)=0$ and $e_{0}-e_{1}=\left(a_{0}-a_{1}\right)+\left(b_{0}-b_{1}\right)+\left(c_{0}-\right.$ $\left.c_{1}\right)+\left(d_{0}-d_{1}\right)=-1$. Thus $L_{4 r, 4 x, 4 t+2,4 b+2}$ is cordial.

Lemma 3.4. The four-leaved rose graph is not cordial if $m, n \equiv 0(\bmod 4)$ and $k, s$ are odd.

Proof. It's easy to verify that the degree of all vertices of $L_{n, m, k, S}$ is even, and $L_{n, m, k, S}$ is an Eulerian graph with size is congruent to $2(\bmod 4)$, then from Cahit's theorem [7], $L_{n, m, k, s}$ is not cordial.

Lemma 3.5. The four-leaved rose graph is cordial if and only if $m, n \equiv 1(\bmod 4)$ and $k, s$ are odd.

Proof. $L_{5,5,3,3}$ is cordial since it's isomorphic to $L_{3,3,5,5}$. Now, we need to study the following cases for $k, s>4$.

Case (1.5): $k, s \equiv 1(\bmod 4)$.

Suppose that $k=4 t+1, s=4 b+1$, where $t, b \geq 1$. We choose the labeling $\left[L_{4 r} 1: L_{4 x}: \bar{S}_{4 t}: L_{4 b}\right]$ for $L_{4 r+1,4 x+1,4 t+1,4 b+1}$. Therefore $x_{0}=2 r, x_{1}=2 r+1$, $a_{0}=2 r+1, a_{1}=2 r, y_{0}=2 x, y_{1}=2 x, b_{0}=2 x+1$, $b_{1}=2 x, z_{0}=2 t, z_{1}=2 t, \quad c_{0}=2 t-1, c_{1}=2 t+2$, $h_{0}=2 b, h_{1}=2 b, d_{0}=2 b+1$ and $d_{1}=2 b$. Hence $v_{0}-v_{1}=\left(x_{0}-x_{1}\right)+\left(y_{0}-y_{1}\right)+\left(z_{0}-z_{1}\right)+\left(h_{0}-\right.$

$\left.h_{1}\right)=-1$ and $e_{0}-e_{1}=\left(a_{0}-a_{1}\right)+\left(b_{0}-b_{1}\right)+\left(c_{0}-\right.$ $\left.c_{1}\right)+\left(d_{0}-d_{1}\right)=0$. Thus $L_{4 r+1,4 x+1,4 t+1,4 b+1}$ is cordial.

Case (2.5): $k, s \equiv 3(\bmod 4)$.

Suppose that $k=4 t+3, s=4 b+3$, where $t, b \geq 1$. We choose the labeling $\left[L_{4 r} 1: L_{4 x}: 0 \bar{L}_{4 t} 1: 1 L_{4 b} 0\right]$ for $L_{4 r+1,4 x+1,4 t+3,4 b+3}$. Therefore $x_{0}=2 r, x_{1}=2 r+1$, $a_{0}=2 r+1, a_{1}=2 r, y_{0}=2 x, y_{1}=2 x, b_{0}=2 x+1$, $b_{1}=2 x, z_{0}=2 t+1, z_{1}=2 t+1, \quad c_{0}=2 t+1, \quad c_{1}=$ $2 t+2, h_{0}=2 b+1, \quad h_{1}=2 b+1, d_{0}=2 b+1$ and $d_{1}=2 b+2$. Hence $v_{0}-v_{1}=\left(x_{0}-x_{1}\right)+\left(y_{0}-y_{1}\right)+$ $\left(z_{0}-z_{1}\right)+\left(h_{0}-h_{1}\right)=-1$ and $e_{0}-e_{1}=\left(a_{0}-a_{1}\right)+$ $\left(b_{0}-b_{1}\right)+\left(c_{0}-c_{1}\right)+\left(d_{0}-d_{1}\right)=0 \quad$. $\quad$ Thus $L_{4 r+1,4 x+1,4 t+3,4 b+3}$ is cordial.

Lemma 3.6. The four-leaved rose graph is not cordial if $m, n \equiv 1(\bmod 4)$ and $k, s$ are even.

Proof. It's easy to verify that the degree of all vertices of 
$L_{n, m, k, s}$ is even, and $L_{n, m, k, s}$ is an Eulerian graph with size is congruent to $2(\bmod 4)$, then from Cahit's theorem [7], $L_{n, m, k, s}$ is not cordial.

Lemma 3.7. The four-leaved rose graph is cordial if $m, n \equiv 2(\bmod 4)$ and $k, s$ are even.

Proof. $L_{6,6,3,3}$ is not cordial since it 's isomorphic to $L_{3,3,6,6}$. Now, we need to study the following cases for $k, s \geq 4$.

Case $(1.7): k, s \equiv 0(\bmod 4)$.

$L_{6,6,4,4}$ is cordial since it 's isomorphic to $L_{4,4,6,6}$.

Case $(2.7): k, s \equiv 2(\bmod 4)$.

Suppose that $k=4 t+2, s=4 b+2$, where $t, b \geq 1$. We choose the labeling $\left[L_{4 r} 10: S_{4 x} 0: \bar{L}_{4 t} 1: 1 \bar{L}_{4 b}\right]$ for $L_{4 r+2,4 x+2,4 t+2,4 b+2}$. Therefore $x_{0}=2 r+1, x_{1}=2 r+1$, $a_{0}=2 r+2, a_{1}=2 r, y_{0}=2 x+1, y_{1}=2 x, b_{0}=2 x$, $b_{1}=2 x+2, z_{0}=2 t, z_{1}=2 t+1, c_{0}=2 t, c_{1}=2 t+2$, $h_{0}=2 b, h_{1}=2 b+1, d_{0}=2 b+2$ and $d_{1}=2 b$. Hence $v_{0}-v_{1}=\left(x_{0}-x_{1}\right)+\left(y_{0}-y_{1}\right)+\left(z_{0}-z_{1}\right)+\left(h_{0}-\right.$ $\left.h_{1}\right)=-1$ and $e_{0}-e_{1}=\left(a_{0}-a_{1}\right)+\left(b_{0}-b_{1}\right)+\left(c_{0}-\right.$ $\left.c_{1}\right)+\left(d_{0}-d_{1}\right)=0$. Thus $L_{4 r+2,4 x+2,4 t+2,4 b+2}$ is cordial.

Lemma 3.8. The four-leaved rose graph is not cordial if $m, n \equiv 2(\bmod 4)$ and $k, s$ are odd.

Proof. It's easy to verify that the degree of all vertices of
$L_{n, m, k, s}$ is even, and $L_{n, m, k, s}$ is an Eulerian graph with size is congruent to $2(\bmod 4)$, then from Cahit's theorem [7], $L_{n, m, k, s}$ is not cordial.

Lemma 3.9. The four-leaved rose graph is cordial if and only if $m, n \equiv 3(\bmod 4)$ and $k, s$ are odd.

Proof. $L_{7,7,3,3}$ is cordial since it's isomorphic to $L_{3,3,7,7}$. Now, we need to study the following cases for $k, s>4$.

Case $(1.9): k, s \equiv 1(\bmod 4)$.

$L_{7,7,5,5}$ is cordial since it 's isomorphic to $L_{5,5,7,7}$.

Case $(2.9): k, s \equiv 3(\bmod 4)$.

Suppose that $k=4 t+3, s=4 b+3$, where $t, b \geq 1$. We choose the labeling $\left[L_{4 r} 101: 10 L_{4 x}: 0 \bar{L}_{4 t} 1: 1 L_{4 b} 0\right]$ for $L_{4 r+3,4 x+3,4 t+3,4 b+3}$. Therefore $x_{0}=2 r+1, x_{1}=2 r+2$, $a_{0}=2 r+1, a_{1}=2 r+2, y_{0}=2 x+1, y_{1}=2 x+1$, $b_{0}=2 x+3, b_{1}=2 x, z_{0}=2 t+1, z_{1}=2 t+1, c_{0}=$ $2 t+1, c_{1}=2 t+2, h_{0}=2 b+1, h_{1}=2 b+1, d_{0}=$ $2 b+1$ and $d_{1}=2 b+2$. Hence $v_{0}-v_{1}=\left(x_{0}-x_{1}\right)+$ $\left(y_{0}-y_{1}\right)+\left(z_{0}-z_{1}\right)+\left(h_{0}-h_{1}\right)=-1$ and $e_{0}-e_{1}=$ $\left(a_{0}-a_{1}\right)+\left(b_{0}-b_{1}\right)+\left(c_{0}-c_{1}\right)+\left(d_{0}-d_{1}\right)=0$. As an example, Figure 1 illustrates $L_{7,7,7,7}$. Thus $L_{4 r+3,4 x+3,4 t+3,4 b+3}$ is cordial.

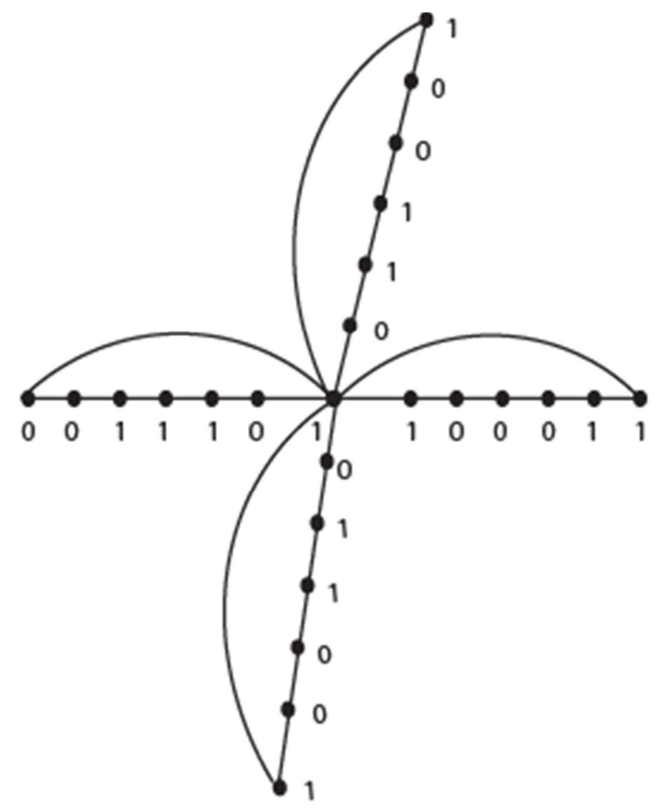

$$
\begin{aligned}
& x_{0}=3, x_{1}=4, a_{0}=3, a_{1}=4, \\
& y_{0}=3, y_{1}=3, b_{0}=5, b_{1}=2, \\
& z_{0}=3, z_{1}=3, c_{0}=3, c_{1}=4 \\
& h_{0}=3, h_{1}=3, d_{0}=3 \\
& \text { and } d_{1}=4 \\
& v_{0}-v_{1}=-1, e_{0}-e_{1}=0 . \\
& L_{7,7,7,7} \text { is cordial }
\end{aligned}
$$

Figure 1. Cordial represention of $L_{7,7,7,7}$.

Lemma 3.10. The four-leaved rose graph is cordial if $m, n \equiv 3(\bmod 4)$ and $k, s$ are even.

Proof. It's easy to verify that the degree of all vertices of $L_{n, m, k, s}$ is even, and $L_{n, m, k, s}$ is an Eulerian graph with size is congruent to $2(\bmod 4)$, then from Cahit's theorem [7], $L_{n, m, k, s}$ is not cordial.

Lemma 3.11 . If $n, m=3$ and $k \equiv i(\bmod 4)$, where $i=0,1,2,3$ then the four-leaved rose graph is cordial except if $(k+s \equiv 0(\bmod 4))$ for all $s \geq 3$.

Proof. Suppose that $s \equiv\{3, j(\bmod 4)\}$, where $j=0,1,2,3$. We need to study the following cases:

Case $(1.11): k \equiv 0(\bmod 4)$.

We choose the labeling [110: $\left.11: 000 \bar{L}_{4 t}: 01\right]$ for $L_{3,3,4 t, 3}$.
Therefore $x_{0}=1, x_{1}=2, a_{0}=1, a_{1}=2, y_{0}=0, y_{1}=2$, $b_{0}=1, b_{1}=2, z_{0}=2 t+3, z_{1}=2 t, c_{0}=2 t+4$, $c_{1}=2 t, h_{0}=1, h_{1}=1, d_{0}=1$ and $d_{1}=2$. Hence $v_{0}-v_{1}=\left(x_{0}-x_{1}\right)+\left(y_{0}-y_{1}\right)+\left(z_{0}-z_{1}\right)+\left(h_{0}-\right.$ $\left.h_{1}\right)=0$ and $e_{0}-e_{1}=\left(a_{0}-a_{1}\right)+\left(b_{0}-b_{1}\right)+\left(c_{0}-\right.$ $\left.c_{1}\right)+\left(d_{0}-d_{1}\right)=1$. Thus $L_{3,3,4 t, 3}$ is cordial. Now, we need to study the following subcases for $s>4$.

Subcase (1.1.11): $s \equiv 1(\bmod 4)$.

Suppose that $s=4 b+1$, where $b \geq 1$. We choose the labeling [110: $\left.11: 000 \bar{L}_{4 t}: S_{4 b}\right]$ for $L_{3,3,4 t, 4 b+1}$. Therefore $x_{0}=1, x_{1}=2, a_{0}=1, a_{1}=2, y_{0}=0, y_{1}=2, b_{0}=1$, $b_{1}=2, z_{0}=2 t+3, z_{1}=2 t, c_{0}=2 t+4, c_{1}=2 t$, $h_{0}=2 b, h_{1}=2 b, d_{0}=2 b-1$ and $d_{1}=2 b+2$. Hence 
$v_{0}-v_{1}=\left(x_{0}-x_{1}\right)+\left(y_{0}-y_{1}\right)+\left(z_{0}-z_{1}\right)+\left(h_{0}-\right.$

$\left.h_{1}\right)=0$ and $e_{0}-e_{1}=\left(a_{0}-a_{1}\right)+\left(b_{0}-b_{1}\right)+\left(c_{0}-\right.$

$\left.c_{1}\right)+\left(d_{0}-d_{1}\right)=-1$. Thus $L_{3,3,4 t, 4 b+1}$ is cordial.

Subcase (2.1.11): $s \equiv 2(\bmod 4)$.

Suppose that $s=4 b+2$, where $b \geq 1$. We choose the labeling [110:11:000 $\left.\bar{L}_{4 t}: 0 S_{4 b}\right]$ for $L_{3,3,4 t, 4 b+2}$. Therefore $x_{0}=1, x_{1}=2, a_{0}=1, a_{1}=2, y_{0}=0, y_{1}=2, b_{0}=1$, $b_{1}=2, z_{0}=2 t+3, z_{1}=2 t, c_{0}=2 t+4, c_{1}=2 t$, $h_{0}=2 b+1, h_{1}=2 b, d_{0}=2 b$ and $d_{1}=2 b+2$. Hence $v_{0}-v_{1}=\left(x_{0}-x_{1}\right)+\left(y_{0}-y_{1}\right)+\left(z_{0}-z_{1}\right)+\left(h_{0}-\right.$

$\left.h_{1}\right)=1$ and $e_{0}-e_{1}=\left(a_{0}-a_{1}\right)+\left(b_{0}-b_{1}\right)+\left(c_{0}-\right.$ $\left.c_{1}\right)+\left(d_{0}-d_{1}\right)=0$. Thus $L_{3,3,4 t, 4 b+2}$ is cordial.

Subcase (3.1.11): $s \equiv 3(\bmod 4)$.

Suppose that $s=4 b+3$, where $b \geq 1$. We choose the labeling [110: 11:000 $\left.\bar{L}_{4 t}: 10 \bar{L}_{4 b}\right]$ for $L_{3,3,4 t, 4 b+3}$. Therefore $x_{0}=1, x_{1}=2, a_{0}=1, a_{1}=2, y_{0}=0, y_{1}=2, b_{0}=1$, $b_{1}=2, z_{0}=2 t+3, z_{1}=2 t, c_{0}=2 t+4, c_{1}=2 t$, $h_{0}=2 b+1, h_{1}=2 b+1, d_{0}=2 b+1$ and $d_{1}=2 b+2$. Hence $\quad v_{0}-v_{1}=\left(x_{0}-x_{1}\right)+\left(y_{0}-y_{1}\right)+\left(z_{0}-z_{1}\right)+$ $\left(h_{0}-h_{1}\right)=0$ and $e_{0}-e_{1}=\left(a_{0}-a_{1}\right)+\left(b_{0}-b_{1}\right)+$ $\left(c_{0}-c_{1}\right)+\left(d_{0}-d_{1}\right)=1$. Thus $L_{3,3,4 t, 4 b+3}$ is cordial.

Case $(2.11): k \equiv 1(\bmod 4)$.

$L_{3,3,5,3}$ is not cordial, Now, we need to study the following subcases for $s \geq 4$.

Subcase (1.2.11): $s \equiv 0(\bmod 4)$.

$L_{3,3,5,4}$ is cordial since it ' $S$ isomorphic to $L_{3,3,4,5}$.

Subcase $(2.2 .11): s \equiv 1(\bmod 4)$.

$L_{3,3,5,5}$ is cordial since it 's isomorphic to $L_{5,5,3,3}$.

Subcase $(3.2 .11): s \equiv 2(\bmod 4)$.

Suppose that $s=4 b+2$, where $b \geq 1$. We choose the labeling [110:10: $\left.\bar{S}_{4 t}: 0 \bar{L}_{4 b}\right]$ for $L_{3,3,4 t+1,4 b+2}$. Therefore $x_{0}=1, x_{1}=2, a_{0}=1, a_{1}=2, y_{0}=1, y_{1}=1, b_{0}=1$, $b_{1}=2, z_{0}=2 t, z_{1}=2 t, c_{0}=2 t+1, c_{1}=2 t, h_{0}=$ $2 b+1, \quad h_{1}=2 b, d_{0}=2 b+2$ and $d_{1}=2 b$. Hence $v_{0}-v_{1}=\left(x_{0}-x_{1}\right)+\left(y_{0}-y_{1}\right)+\left(z_{0}-z_{1}\right)+\left(h_{0}-\right.$ $\left.h_{1}\right)=0$ and $e_{0}-e_{1}=\left(a_{0}-a_{1}\right)+\left(b_{0}-b_{1}\right)+\left(c_{0}-\right.$ $\left.c_{1}\right)+\left(d_{0}-d_{1}\right)=1$. Thus $L_{3,3,4 t+1,4 b+2}$ is cordial.

Case $(3.11): k \equiv 2(\bmod 4)$.

We choose the labeling [110: 10: $\left.L_{4 t} 0: 01\right]$ for $L_{3,3,4 t+2,3}$. Therefore $x_{0}=1, x_{1}=2, a_{0}=1, a_{1}=2, y_{0}=1, y_{1}=1$, $b_{0}=1, \quad b_{1}=2, z_{0}=2 t+1, z_{1}=2 t, c_{0}=2 t+2$, $c_{1}=2 t, h_{0}=1, h_{1}=1, d_{0}=1$ and $d_{1}=2$. Hence $v_{0}-v_{1}=\left(x_{0}-x_{1}\right)+\left(y_{0}-y_{1}\right)+\left(z_{0}-z_{1}\right)+\left(h_{0}-\right.$ $\left.h_{1}\right)=0$ and $e_{0}-e_{1}=\left(a_{0}-a_{1}\right)+\left(b_{0}-b_{1}\right)+\left(c_{0}-\right.$ $\left.c_{1}\right)+\left(d_{0}-d_{1}\right)=-1$. Thus $L_{3,3,4 t+2,3}$ is cordial. Now, we need to study the following subcases for $s \geq 4$.

Subcase (1.3.11): $s \equiv 0(\bmod 4)$.

$L_{3,3,6,4}$ is cordial since it 's isomorphic to $L_{3,3,4,6}$.

Subcase (2.3.11): $s \equiv 1(\bmod 4)$.

$L_{3,3,6,5}$ is cordial since it 's isomorphic to $L_{3,3,5,6}$.

Subcase (3.3.11): $s \equiv 3(\bmod 4)$.

Suppose that $s=4 b+3$, where $b \geq 1$. We choose the labeling [110: 10: $L_{4 t} 0: 01 S_{4 b}$ ] for $L_{3,3,4 t+2,4 b+3}$. Therefore $x_{0}=1, x_{1}=2, a_{0}=1, a_{1}=2, y_{0}=1, y_{1}=1, b_{0}=1$, $b_{1}=2, z_{0}=2 t+1, z_{1}=2 t, c_{0}=2 t+2, c_{1}=2 t$, $h_{0}=2 b+1, h_{1}=2 b+1, d_{0}=2 b+1$ and $d_{1}=2 b+2$. Hence $\quad v_{0}-v_{1}=\left(x_{0}-x_{1}\right)+\left(y_{0}-y_{1}\right)+\left(z_{0}-z_{1}\right)+$ $\left(h_{0}-h_{1}\right)=0 \quad$ and $\quad e_{0}-e_{1}=\left(a_{0}-a_{1}\right)+\left(b_{0}-b_{1}\right)+$ $\left(c_{0}-c_{1}\right)+\left(d_{0}-d_{1}\right)=-1$. Thus $L_{3,3,4 t+2,4 b+3}$ is cordial.

Case $(4.11): k \equiv 3(\bmod 4)$.

We choose the labeling [110:11:10 $\left.\bar{L}_{4 t}: 00\right]$ for $L_{3,3,4 t+3,3}$. Therefore $x_{0}=1, x_{1}=2, a_{0}=1, a_{1}=2, y_{0}=0, y_{1}=2$, $b_{0}=1, b_{1}=2, z_{0}=2 t+1, z_{1}=2 t+1, c_{0}=2 t+1$, $c_{1}=2 t+2, h_{0}=2, h_{1}=0, d_{0}=3$ and $d_{1}=0$. Hence $v_{0}-v_{1}=\left(x_{0}-x_{1}\right)+\left(y_{0}-y_{1}\right)+\left(z_{0}-z_{1}\right)+\left(h_{0}-\right.$ $\left.h_{1}\right)=-1$ and $e_{0}-e_{1}=\left(a_{0}-a_{1}\right)+\left(b_{0}-b_{1}\right)+\left(c_{0}-\right.$ $\left.c_{1}\right)+\left(d_{0}-d_{1}\right)=0$. Thus $L_{3,3,4 t+3,3}$ is cordial. Now, we need to study the following subcases for $s \geq 4$.

Subcase (1.4.11): $s \equiv 0(\bmod 4)$.

$L_{3,3,7,4}$ is cordial since it 's isomorphic to $L_{3,3,4,7}$.

Subcase $(2.4 .11): s \equiv 2(\bmod 4)$.

$L_{3,3,7,6}$ is cordial since it 's isomorphic to $L_{3,3,6,7}$.

Subcase (3.4.11): $s \equiv 3(\bmod 4)$.

$L_{3,3,7,7}$ is cordial since it 's isomorphic to $L_{7,7,3,3}$.

Lemma 3.12 . If $n, m=3$ and $k \equiv i(\bmod 4)$, where $i=0,1,2,3$ then the four-leaved rose graph is not cordial if $(k+s \equiv 0(\bmod 4))$ for all $s \geq 3$.

Proof. It's easy to verify that the degree of all vertices of $L_{n, m, k, s}$ is even, and $L_{n, m, k, s}$ is an Eulerian graph with size is congruent to $2(\bmod 4)$, then from Cahit's theorem [7], $L_{n, m, k, s}$ is not cordial.

Lemma 3.13. If $n, m \equiv 0(\bmod 4)$ and $k \equiv i(\bmod 4)$, where $i=0,1,2,3$ then the four-leaved rose graph is cordial except if $(k+s \equiv 2(\bmod 4))$ for all $s \geq 3$.

Proof. Suppose that $s \equiv\{3, j(\bmod 4)\}$, where $j=0,1,2,3$.

We need to study the following cases:

Case $(1.13): k \equiv 0(\bmod 4)$.

We choose the labeling $\left[L_{4 r}: 101 L_{4 x}: L_{4 t} 100: 10\right]$ for $L_{4 r, 4 x, 4 t, 3}$. Therefore $x_{0}=2 r, x_{1}=2 r, a_{0}=2 r, a_{1}=2 r$, $y_{0}=2 x+1, y_{1}=2 x+2, b_{0}=2 x+2, b_{1}=2 x+2$, $z_{0}=2 t+2, z_{1}=2 t+1, \quad c_{0}=2 t+2, \quad c_{1}=2 t+2$, $h_{0}=1, h_{1}=1, d_{0}=1$ and $d_{1}=2$. Hence $v_{0}-v_{1}=$ $\left(x_{0}-x_{1}\right)+\left(y_{0}-y_{1}\right)+\left(z_{0}-z_{1}\right)+\left(h_{0}-h_{1}\right)=0 \quad$ and $e_{0}-e_{1}=\left(a_{0}-a_{1}\right)+\left(b_{0}-b_{1}\right)+\left(c_{0}-c_{1}\right)+\left(d_{0}-d_{1}\right)=$ -1 . Thus $L_{4 r, 4 x, 4 t, 3}$ is cordial. Now, we need to study the following subcases for $s \geq 4$.

Subcase (1.1.13): $s \equiv 0(\bmod 4)$.

$L_{4,4,4,4}$ is cordial since it 's isomorphic to $L_{4,4,4,4}$.

Subcase (2.1.13): $s \equiv 1(\bmod 4)$.

Suppose that $s=4 b+1$, where $b \geq 1$. We choose the labeling $\left[L_{4 r}: 101 L_{4 x}: L_{4 t} 100: \bar{L}_{4 b}\right]$ for $L_{4 r, 4 x, 4 t, 4 b+1}$. Therefore $x_{0}=2 r, x_{1}=2 r, a_{0}=2 r, a_{1}=2 r, y_{0}=2 x+$ $1, y_{1}=2 x+2, b_{0}=2 x+2, b_{1}=2 x+2, z_{0}=2 t+2$, $z_{1}=2 t+1, c_{0}=2 t+2, c_{1}=2 t+2, h_{0}=2 b, h_{1}=2 b$, $d_{0}=2 b+1$ and $d_{1}=2 b$. Hence $v_{0}-v_{1}=\left(x_{0}-x_{1}\right)+$ $\left(y_{0}-y_{1}\right)+\left(z_{0}-z_{1}\right)+\left(h_{0}-h_{1}\right)=0$ and $e_{0}-e_{1}=$ $\left(a_{0}-a_{1}\right)+\left(b_{0}-b_{1}\right)+\left(c_{0}-c_{1}\right)+\left(d_{0}-d_{1}\right)=1$. Thus $L_{4 r, 4 x, 4 t, 4 b+1}$ is cordial.

Subcase (3.1.13): $s \equiv 3(\bmod 4)$.

Suppose that $s=4 b+3$, where $b \geq 1$. We choose the labeling $\left[L_{4 r}: 101 L_{4 x}: L_{4 t} 100: 01 \bar{L}_{4 b}\right]$ for $L_{4 r, 4 x, 4 t, 4 b+3}$. Therefore $x_{0}=2 r, x_{1}=2 r, a_{0}=2 r, a_{1}=2 r, y_{0}=2 x+$ $1, y_{1}=2 x+2, b_{0}=2 x+2, b_{1}=2 x+2, z_{0}=2 t+2$, $z_{1}=2 t+1, \quad c_{0}=2 t+2, \quad c_{1}=2 t+2, \quad h_{0}=2 b+1$, 
$h_{1}=2 b+1, d_{0}=2 b+1$ and $d_{1}=2 b+2$. Hence $v_{0}-v_{1}=\left(x_{0}-x_{1}\right)+\left(y_{0}-y_{1}\right)+\left(z_{0}-z_{1}\right)+\left(h_{0}-\right.$ $\left.h_{1}\right)=0$ and $e_{0}-e_{1}=\left(a_{0}-a_{1}\right)+\left(b_{0}-b_{1}\right)+\left(c_{0}-\right.$ $\left.c_{1}\right)+\left(d_{0}-d_{1}\right)=-1$. Thus $L_{4 r, 4 x, 4 t, 4 b+3}$ is cordial.

Case $(2.13): k \equiv 1(\bmod 4)$.

We choose the labeling $\left[L_{4 r}: 101 L_{4 x}: \bar{L}_{4 t}: 10\right]$ for $L_{4 r, 4 x, 4 t+1,3}$. Therefore $x_{0}=2 r, x_{1}=2 r, a_{0}=2 r$, $a_{1}=2 r, y_{0}=2 x+1, y_{1}=2 x+2, b_{0}=2 x+2, b_{1}=$ $2 x+2, z_{0}=2 t, z_{1}=2 t, c_{0}=2 t+1, c_{1}=2 t, h_{0}=1$, $h_{1}=1, d_{0}=1$ and $d_{1}=2$. Hence $v_{0}-v_{1}=\left(x_{0}-x_{1}\right)+$ $\left(y_{0}-y_{1}\right)+\left(z_{0}-z_{1}\right)+\left(h_{0}-h_{1}\right)=-1$ and $e_{0}-e_{1}=$ $\left(a_{0}-a_{1}\right)+\left(b_{0}-b_{1}\right)+\left(c_{0}-c_{1}\right)+\left(d_{0}-d_{1}\right)=0$. Thus $L_{4 r, 4 x, 4 t+1,3}$ is cordial. Now, we need to study the following subcases for $s \geq 4$.

Subcase (1.2.13): $s \equiv 0(\bmod 4)$.

$L_{4,4,5,4}$ is cordial since it's isomorphic to $L_{4,4,4,5}$.

Subcase (2.2.13): $s \equiv 2(\bmod 4)$.

Suppose that $s=4 b+2$, where $b \geq 1$. We choose the labeling $\left[L_{4 r}: 101 L_{4 x}: \bar{L}_{4 t}: 0 \bar{L}_{4 b}\right]$ for $L_{4 r, 4 x, 4 t+1,4 b+2}$. Therefore $x_{0}=2 r, x_{1}=2 r, a_{0}=2 r, a_{1}=2 r, y_{0}=2 x+$ $1, y_{1}=2 x+2, b_{0}=2 x+2, b_{1}=2 x+2, z_{0}=2 t$, $z_{1}=2 t, \quad c_{0}=2 t+1, c_{1}=2 t, h_{0}=2 b+1, h_{1}=2 b$, $d_{0}=2 b$ and $d_{1}=2 b+2$. Hence $v_{0}-v_{1}=\left(x_{0}-x_{1}\right)+$ $\left(y_{0}-y_{1}\right)+\left(z_{0}-z_{1}\right)+\left(h_{0}-h_{1}\right)=0$ and $e_{0}-e_{1}=$ $\left(a_{0}-a_{1}\right)+\left(b_{0}-b_{1}\right)+\left(c_{0}-c_{1}\right)+\left(d_{0}-d_{1}\right)=-1$. Thus $L_{4 r, 4 x, 4 t+1,4 b+2}$ is cordial.

Subcase $(3.2 .13): s \equiv 3(\bmod 4)$.

Suppose that $s=4 b+3$, where $b \geq 1$. We choose the labeling $\left[L_{4 r}: 101 L_{4 x}: \bar{L}_{4 t}: 10 \bar{L}_{4 b}\right]$ for $L_{4 r, 4 x, 4 t+1,4 b+3}$. Therefore $x_{0}=2 r, x_{1}=2 r, a_{0}=2 r, a_{1}=2 r, y_{0}=2 x+$ $1, y_{1}=2 x+2, b_{0}=2 x+2, b_{1}=2 x+2, z_{0}=2 t$, $z_{1}=2 t, c_{0}=2 t+1, c_{1}=2 t, h_{0}=2 b+1, h_{1}=2 b+1$, $d_{0}=2 b+1$ and $d_{1}=2 b+2$. Hence $v_{0}-v_{1}=\left(x_{0}-\right.$ $\left.x_{1}\right)+\left(y_{0}-y_{1}\right)+\left(z_{0}-z_{1}\right)+\left(h_{0}-h_{1}\right)=-1 \quad$ and $e_{0}-e_{1}=\left(a_{0}-a_{1}\right)+\left(b_{0}-b_{1}\right)+\left(c_{0}-c_{1}\right)+\left(d_{0}-d_{1}\right)=$ 0 . Thus $L_{4 r, 4 x, 4 t+1,4 b+3}$ is cordial.

Case $(3.13): k \equiv 2(\bmod 4)$.

We choose the labeling $\left[L_{4 r}: 101 L_{4 x}: \bar{L}_{4 t} 0: 10\right]$ for $L_{4 r, 4 x, 4 t+2,3}$. Therefore $x_{0}=2 r, x_{1}=2 r, a_{0}=2 r$, $a_{1}=2 r, y_{0}=2 x+1, y_{1}=2 x+2, b_{0}=2 x+2, b_{1}=$ $2 x+2, z_{0}=2 t+1, z_{1}=2 t, c_{0}=2 t+2, c_{1}=2 t$, $h_{0}=1, h_{1}=1, d_{0}=1$ and $d_{1}=2$. Hence $v_{0}-v_{1}=$ $\left(x_{0}-x_{1}\right)+\left(y_{0}-y_{1}\right)+\left(z_{0}-z_{1}\right)+\left(h_{0}-h_{1}\right)=0 \quad$ and $e_{0}-e_{1}=\left(a_{0}-a_{1}\right)+\left(b_{0}-b_{1}\right)+\left(c_{0}-c_{1}\right)+\left(d_{0}-d_{1}\right)=$ 1 . Thus $L_{4 r, 4 x, 4 t+2,3}$ is cordial. Now, we need to study the following subcases for $s>4$.

Subcase $(1.3 .13): s \equiv 1(\bmod 4)$.

$L_{4,4,6,5}$ is cordial since it 's isomorphic to $L_{4,4,5,6}$.

Subcase $(2.3 .13): s \equiv 2(\bmod 4)$.

$L_{4,4,6,6}$ is cordial since it 's isomorphic to $L_{6,6,4,4}$.

Subcase $(3.3 .13): s \equiv 3(\bmod 4)$.

Suppose that $s=4 b+3$, where $b \geq 1$. We choose the labeling $\left[L_{4 r}: 101 L_{4 x}: \bar{L}_{4 t} 0: 10 \bar{L}_{4 b}\right]$ for $L_{4 r, 4 x, 4 t+2,4 b+3}$. Therefore $x_{0}=2 r, x_{1}=2 r, a_{0}=2 r, a_{1}=2 r, y_{0}=2 x+$ $1, y_{1}=2 x+2, b_{0}=2 x+2, b_{1}=2 x+2, z_{0}=2 t+1$, $z_{1}=2 t, c_{0}=2 t+2, c_{1}=2 t, h_{0}=2 b+1, h_{1}=2 b+1$, $d_{0}=2 b+1$ and $d_{1}=2 b+2$. Hence $v_{0}-v_{1}=\left(x_{0}-\right.$ $\left.x_{1}\right)+\left(y_{0}-y_{1}\right)+\left(z_{0}-z_{1}\right)+\left(h_{0}-h_{1}\right)=0$ and $e_{0}-$ $e_{1}=\left(a_{0}-a_{1}\right)+\left(b_{0}-b_{1}\right)+\left(c_{0}-c_{1}\right)+\left(d_{0}-d_{1}\right)=1$.

Thus $L_{4 r, 4 x, 4 t+2,4 b+3}$ is cordial.

Case (4.13): $k \equiv 3(\bmod 4)$.

$L_{4,4,7,3}$ is not cordial. Now, we need to study the following subcases for $s \geq 4$.

Subcase (1.4.13): $s \equiv 0(\bmod 4)$.

$L_{4,4,7,4}$ is cordial since it 's isomorphic to $L_{4,4,4,7}$.

Subcase $(2.4 .13): s \equiv 1(\bmod 4)$.

$L_{4,4,7,5}$ is cordial since it 's isomorphic to $L_{4,4,5,7}$.

Subcase (3.4.13): $s \equiv 2(\bmod 4)$.

$L_{4,4,7,6}$ is cordial since it 's isomorphic to $L_{4,4,6,7}$.

Lemma 3.14 . If $n, m \equiv 0(\bmod 4)$ and $k \equiv i(\bmod 4)$, where $i=0,1,2,3$ then the four-leaved rose graph is not cordial where $(k+s \equiv 2(\bmod 4))$ for all $s \geq 3$.

Proof. It's easy to verify that the degree of all vertices of $L_{n, m, k, s}$ is even, and $L_{n, m, k, s}$ is an Eulerian graph with size is congruent to $2(\bmod 4)$, then from Cahit's theorem [7], $L_{n, m, k, s}$ is not cordial.

Lemma 3.15. If $n, m \equiv 1(\bmod 4)$ and $k \equiv i(\bmod 4)$, where $i=0,1,2,3$ then the four-leaved rose graph is cordial except if $(k+s \equiv 0(\bmod 4))$ for all $s \geq 3$.

Proof. Suppose that $s \equiv\{3, j(\bmod 4)\}$, where $j=0,1,2,3$. We need to study the following cases:

Case $(1.15): k \equiv 0(\bmod 4)$.

We choose the labeling $\left[L_{4 r} 1: S_{4 x}: 100 \bar{L}_{4 t}: 10\right]$ for $L_{4 r+1,4 x+1,4 t, 3}$. Therefore $x_{0}=2 r, x_{1}=2 r+1, a_{0}=2 r+$ $1, a_{1}=2 r, y_{0}=2 x, y_{1}=2 x, b_{0}=2 x+1, b_{1}=2 x$, $z_{0}=2 t+2, \quad z_{1}=2 t+1, \quad c_{0}=2 t+2, \quad c_{1}=2 t+2$, $h_{0}=1, h_{1}=1, d_{0}=1$ and $d_{1}=2$. Hence $v_{0}-v_{1}=$ $\left(x_{0}-x_{1}\right)+\left(y_{0}-y_{1}\right)+\left(z_{0}-z_{1}\right)+\left(h_{0}-h_{1}\right)=0 \quad$ and $e_{0}-e_{1}=\left(a_{0}-a_{1}\right)+\left(b_{0}-b_{1}\right)+\left(c_{0}-c_{1}\right)+\left(d_{0}-d_{1}\right)=$ 1 . Thus $L_{4 r+1,4 x+1,4 t, 3}$ is cordial. Now, we need to study the following subcases for $s>4$.

Subcase (1.1.15): $s \equiv 1(\bmod 4)$.

Suppose that $s=4 b+1$, where $b \geq 1$. We choose the labeling $\left[L_{4 r} 1: S_{4 x}: 100 \bar{L}_{4 t}: \bar{S}_{4 b}\right]$ for $L_{4 r+1,4 x+1,4 t, 4 b+1}$. Therefore $x_{0}=2 r, x_{1}=2 r+1, a_{0}=2 r+1, a_{1}=2 r, y_{0}=2 x, y_{1}=2 x$, $b_{0}=2 x+1, b_{1}=2 x, z_{0}=2 t+2, z_{1}=2 t+1, c_{0}=2 t+2$, $c_{1}=2 t+2, h_{0}=2 b, h_{1}=2 b, d_{0}=2 b-1$ and $d_{1}=2 b+2$. Hence $\quad v_{0}-v_{1}=\left(x_{0}-x_{1}\right)+\left(y_{0}-y_{1}\right)+\left(z_{0}-z_{1}\right)+\left(h_{0}-\right.$ $\left.h_{1}\right)=0 \quad$ and $\quad e_{0}-e_{1}=\left(a_{0}-a_{1}\right)+\left(b_{0}-b_{1}\right)+\left(c_{0}-c_{1}\right)+$ $\left(d_{0}-d_{1}\right)=-1$. Thus $L_{4 r+1,4 x+1,4 t, 4 b+1}$ is cordial.

Subcase $(2.1 .15): s \equiv 2(\bmod 4)$.

Suppose that $s=4 b+2$, where $b \geq 1$. We choose the labeling $\left[L_{4 r} 1: S_{4 x}: 100 \bar{L}_{4 t}: 0 \bar{S}_{4 b}\right]$ for $L_{4 r+1,4 x+1,4 t, 4 b+2}$. Therefore $x_{0}=2 r, x_{1}=2 r+1, a_{0}=2 r+1, a_{1}=2 r$, $y_{0}=2 x, y_{1}=2 x, b_{0}=2 x+1, b_{1}=2 x, z_{0}=2 t+2$, $z_{1}=2 t+1, \quad c_{0}=2 t+2, \quad c_{1}=2 t+2, \quad h_{0}=2 b+1$, $h_{1}=2 b, d_{0}=2 b$ and $d_{1}=2 b+2$. Hence $v_{0}-v_{1}=$ $\left(x_{0}-x_{1}\right)+\left(y_{0}-y_{1}\right)+\left(z_{0}-z_{1}\right)+\left(h_{0}-h_{1}\right)=1 \quad$ and $e_{0}-e_{1}=\left(a_{0}-a_{1}\right)+\left(b_{0}-b_{1}\right)+\left(c_{0}-c_{1}\right)+\left(d_{0}-d_{1}\right)=$ 0 . Thus $L_{4 r+1,4 x+1,4 t, 4 b+2}$ is cordial.

Subcase (3.1.15): $s \equiv 3(\bmod 4)$.

Suppose that $s=4 b+3$, where $b \geq 1$. We choose the labeling $\left[L_{4 r} 1: S_{4 x}: 100 \bar{L}_{4 t}: 10 \bar{S}_{4 b}\right]$ for $L_{4 r+1,4 x+1,4 t, 4 b+3}$. Therefore $x_{0}=2 r, x_{1}=2 r+1, a_{0}=2 r+1, a_{1}=2 r$, 
$y_{0}=2 x, y_{1}=2 x, b_{0}=2 x+1, b_{1}=2 x, z_{0}=2 t+2$, $z_{1}=2 t+1, \quad c_{0}=2 t+2, \quad c_{1}=2 t+2, \quad h_{0}=2 b+1$, $h_{1}=2 b+1, d_{0}=2 b+1$ and $d_{1}=2 b+2$. Hence $v_{0}-v_{1}=\left(x_{0}-x_{1}\right)+\left(y_{0}-y_{1}\right)+\left(z_{0}-z_{1}\right)+\left(h_{0}-\right.$ $\left.h_{1}\right)=0$ and $e_{0}-e_{1}=\left(a_{0}-a_{1}\right)+\left(b_{0}-b_{1}\right)+\left(c_{0}-\right.$ $\left.c_{1}\right)+\left(d_{0}-d_{1}\right)=1$. Thus $L_{4 r+1,4 x+1,4 t, 4 b+3}$ is cordial.

Case $(2.15): k \equiv 1(\bmod 4)$.

$L_{5,5,5,3}$ is cordial. Now, we need to study the following subcases for $s \geq 4$.

Subcase (1.2.15): $s \equiv 0(\bmod 4)$.

$L_{5,5,5,4}$ is cordial since it 's isomorphic to $L_{5,5,4,5}$.

Subcase (2.2.15): $s \equiv 1(\bmod 4)$.

$L_{5,5,5,5}$ is cordial since it 's isomorphic to $L_{5,5,5,5}$.

Subcase $(3.2 .15): s \equiv 2(\bmod 4)$.

Suppose that $s=4 b+2$, where $b \geq 1$. We choose the labeling $\left[L_{4 r} 1: \bar{S}_{4 x}: L_{4 t}: 0 L_{4 b}\right]$ for $L_{4 r+1,4 x+1,4 t+1,4 b+2}$. Therefore $x_{0}=2 r, x_{1}=2 r+1, a_{0}=2 r+1, a_{1}=2 r$, $y_{0}=2 x, y_{1}=2 x, b_{0}=2 x-1, b_{1}=2 x+2, z_{0}=2 t$, $z_{1}=2 t, \quad c_{0}=2 t+1, c_{1}=2 t, h_{0}=2 b+1, h_{1}=2 b$, $d_{0}=2 b+2$ and $d_{1}=2 b$. Hence $v_{0}-v_{1}=\left(x_{0}-x_{1}\right)+$ $\left(y_{0}-y_{1}\right)+\left(z_{0}-z_{1}\right)+\left(h_{0}-h_{1}\right)=0$ and $e_{0}-e_{1}=$ $\left(a_{0}-a_{1}\right)+\left(b_{0}-b_{1}\right)+\left(c_{0}-c_{1}\right)+\left(d_{0}-d_{1}\right)=1$. Thus $L_{4 r+1,4 x+1,4 t+1,4 b+2}$ is cordial.

Case $(3.15): k \equiv 2(\bmod 4)$.

We choose the labeling $\left[L_{4 r} 1: L_{4 x}: \bar{S}_{4 t} 0: 10\right]$ for $L_{4 r+1,4 x+1,4 t+2,3}$. Therefore $x_{0}=2 r, x_{1}=2 r+1, a_{0}=$ $2 r+1, a_{1}=2 r, y_{0}=2 x, y_{1}=2 x, b_{0}=2 x+1, b_{1}=2 x$ $z_{0}=2 t+1, z_{1}=2 t, \quad c_{0}=2 t, \quad c_{1}=2 t+2, \quad h_{0}=1$, $h_{1}=1, d_{0}=1$ and $d_{1}=2$. Hence $v_{0}-v_{1}=\left(x_{0}-x_{1}\right)+$ $\left(y_{0}-y_{1}\right)+\left(z_{0}-z_{1}\right)+\left(h_{0}-h_{1}\right)=0$ and $e_{0}-e_{1}=$ $\left(a_{0}-a_{1}\right)+\left(b_{0}-b_{1}\right)+\left(c_{0}-c_{1}\right)+\left(d_{0}-d_{1}\right)=-1$. Thus $L_{4 r+1,4 x+1,4 t+2,3}$ is cordial. Now, we need to study the following subcases for $s \geq 4$.

Subcase (1.3.15): $s \equiv 0(\bmod 4)$.

$L_{5,5,6,4}$ is cordial since it 's isomorphic to $L_{5,5,4,6}$.

Subcase (2.3.15): $s \equiv 1(\bmod 4)$.

$L_{5,5,6,5}$ is cordial since it 's isomorphic to $L_{5,5,5,6}$.

Subcase $(3.3 .15): s \equiv 3(\bmod 4)$.

Suppose that $s=4 b+3$, where $b \geq 1$. We choose the labeling $\left[L_{4 r} 1: L_{4 x}: \bar{S}_{4 t} 0: 10 \bar{L}_{4 b}\right]$ for $L_{4 r+1,4 x+1,4 t+2,4 b+3}$. Therefore $x_{0}=2 r, x_{1}=2 r+1, a_{0}=2 r+1, a_{1}=2 r$, $y_{0}=2 x, y_{1}=2 x, b_{0}=2 x+1, b_{1}=2 x, z_{0}=2 t+1$, $z_{1}=2 t, c_{0}=2 t, c_{1}=2 t+2, h_{0}=2 b+1, h_{1}=2 b+1$, $d_{0}=2 b+1$ and $d_{1}=2 b+2$. Hence $v_{0}-v_{1}=\left(x_{0}-\right.$ $\left.x_{1}\right)+\left(y_{0}-y_{1}\right)+\left(z_{0}-z_{1}\right)+\left(h_{0}-h_{1}\right)=0$ and $e_{0}-$ $e_{1}=\left(a_{0}-a_{1}\right)+\left(b_{0}-b_{1}\right)+\left(c_{0}-c_{1}\right)+\left(d_{0}-d_{1}\right)=-1$. Thus $L_{4 r+1,4 x+1,4 t+2,4 b+3}$ is cordial.

Case $(4.15): k \equiv 3(\bmod 4)$.

We choose the labeling $\left[L_{4 r} 1: L_{4 x}: \bar{S}_{4 t} 01: 10\right]$ for $L_{4 r+1,4 x+1,4 t+3,3}$. Therefore $x_{0}=2 r, x_{1}=2 r+1, a_{0}=$ $2 r+1, a_{1}=2 r, y_{0}=2 x, y_{1}=2 x, b_{0}=2 x+1, b_{1}=2 x$ $z_{0}=2 t+1, \quad z_{1}=2 t+1, \quad c_{0}=2 t+1, \quad c_{1}=2 t+2$, $h_{0}=1, h_{1}=1, d_{0}=1$ and $d_{1}=2$. Hence $v_{0}-v_{1}=$ $\left(x_{0}-x_{1}\right)+\left(y_{0}-y_{1}\right)+\left(z_{0}-z_{1}\right)+\left(h_{0}-h_{1}\right)=-1 \quad$ and $e_{0}-e_{1}=\left(a_{0}-a_{1}\right)+\left(b_{0}-b_{1}\right)+\left(c_{0}-c_{1}\right)+\left(d_{0}-d_{1}\right)=$ 0 . Thus $L_{4 r+1,4 x+1,4 t+3,3}$ is cordial. Now, we need to study the following subcases for $s \geq 4$.
Subcase (1.4.15): $s \equiv 0(\bmod 4)$.

$L_{5,5,7,4}$ is cordial since it 's isomorphic to $L_{5,5,4,7}$.

Subcase $(2.4 .15): s \equiv 2(\bmod 4)$.

$L_{5,5,7,6}$ is cordial since it 's isomorphic to $L_{5,5,6,7}$.

Subcase $(3.4 .15): s \equiv 3(\bmod 4)$.

$L_{5,5,7,7}$ is cordial since it 's isomorphic to $L_{7,7,5,5}$.

Lemma 3.16 . If $n, m \equiv 1(\bmod 4)$ and $k \equiv i(\bmod 4)$, where $i=0,1,2,3$ then the four-leaved rose graph is not cordial if $(k+s \equiv 0(\bmod 4))$ for all $s \geq 3$.

Proof. It's easy to verify that the degree of all vertices of $L_{n, m, k, s}$ is even, and $L_{n, m, k, s}$ is an Eulerian graph with size is congruent to $2(\bmod 4)$, then from Cahit's theorem [7], $L_{n, m, k, s}$ is not cordial.

Lemma 3.17 . If $n, m \equiv 2(\bmod 4)$ and $k \equiv i(\bmod 4)$, where $i=0,1,2,3$ then the four-leaved rose graph is cordial except if $(k+s \equiv 2(\bmod 4))$ for all $s \geq 3$.

Proof. Suppose that $s \equiv\{3, j(\bmod 4)\}$, where $j=0,1,2,3$. We need to study the following cases:

Case $(1.17): k \equiv 0(\bmod 4)$.

We choose the labeling $\left[\bar{L}_{4 r} 10: L_{4 x} 1: \bar{L}_{4 t} 100: 10\right]$ for $L_{4 r+2,4 x+2,4 t, 3}$. Therefore $x_{0}=2 r+1, x_{1}=2 r+1$, $a_{0}=2 r, a_{1}=2 r+2, y_{0}=2 x, y_{1}=2 x+1, b_{0}=2 x+2$, $b_{1}=2 x, z_{0}=2 t+2, z_{1}=2 t+1, c_{0}=2 t+2, c_{1}=$ $2 t+2, h_{0}=1, h_{1}=1, d_{0}=1$ and $d_{1}=2$. Hence $v_{0}-v_{1}=\left(x_{0}-x_{1}\right)+\left(y_{0}-y_{1}\right)+\left(z_{0}-z_{1}\right)+\left(h_{0}-\right.$ $\left.h_{1}\right)=0$ and $e_{0}-e_{1}=\left(a_{0}-a_{1}\right)+\left(b_{0}-b_{1}\right)+\left(c_{0}-\right.$ $\left.c_{1}\right)+\left(d_{0}-d_{1}\right)=-1$. Thus $L_{4 r+2,4 x+2,4 t, 3}$ is cordial. Now, we need to study the following subcases for $s \geq 4$.

Subcase (1.1.17): $s \equiv 0(\bmod 4)$.

$L_{6,6,4,4}$ is cordial since it 's isomorphic to $L_{4,4,6,6}$.

Subcase (2.1.17): $s \equiv 1(\bmod 4)$.

Suppose that $s=4 b+1$, where $b \geq 1$. We choose the labeling $\left[\bar{L}_{4 r} 10: L_{4 x} 1: \bar{L}_{4 t} 100: \bar{L}_{4 b}\right]$ for $L_{4 r+2,4 x+2,4 t, 4 b+1}$. Therefore $x_{0}=2 r+1, x_{1}=2 r+1, a_{0}=2 r, a_{1}=2 r+$ $2, y_{0}=2 x, y_{1}=2 x+1, b_{0}=2 x+2, b_{1}=2 x, z_{0}=$ $2 t+2, z_{1}=2 t+1, c_{0}=2 t+2, c_{1}=2 t+2, h_{0}=2 b$, $h_{1}=2 b, d_{0}=2 b+1$ and $d_{1}=2 b$. Hence $v_{0}-v_{1}=$ $\left(x_{0}-x_{1}\right)+\left(y_{0}-y_{1}\right)+\left(z_{0}-z_{1}\right)+\left(h_{0}-h_{1}\right)=0 \quad$ and $e_{0}-e_{1}=\left(a_{0}-a_{1}\right)+\left(b_{0}-b_{1}\right)+\left(c_{0}-c_{1}\right)+\left(d_{0}-d_{1}\right)=$

1. Thus $L_{4 r+2,4 x+2,4 t, 4 b+1}$ is cordial.

Subcase (3.1.17): $s \equiv 3(\bmod 4)$.

Suppose that $s=4 b+3$, where $b \geq 1$. We choose the labeling $\left[\bar{L}_{4 r} 10: L_{4 x} 1: \bar{L}_{4 t} 100: 10 \bar{L}_{4 b}\right]$ for $L_{4 r+2,4 x+2,4 t, 4 b+3}$. Therefore $x_{0}=2 r+1, x_{1}=2 r+1, a_{0}=2 r, a_{1}=2 r+$ $2, y_{0}=2 x, y_{1}=2 x+1, b_{0}=2 x+2, b_{1}=2 x, z_{0}=$ $2 t+2, z_{1}=2 t+1, c_{0}=2 t+2, c_{1}=2 t+2, h_{0}=2 b+$ $1, h_{1}=2 b+1, d_{0}=2 b+1$ and $d_{1}=2 b+2$. Hence $v_{0}-v_{1}=\left(x_{0}-x_{1}\right)+\left(y_{0}-y_{1}\right)+\left(z_{0}-z_{1}\right)+\left(h_{0}-\right.$ $\left.h_{1}\right)=0$ and $e_{0}-e_{1}=\left(a_{0}-a_{1}\right)+\left(b_{0}-b_{1}\right)+\left(c_{0}-\right.$ $\left.c_{1}\right)+\left(d_{0}-d_{1}\right)=-1$. Thus $L_{4 r+2,4 x+2,4 t, 4 b+3}$ is cordial.

Case $(2.17): k \equiv 1(\bmod 4)$.

We choose the labeling $\left[\bar{L}_{4 r} 10: L_{4 x} 1: L_{4 t}: 10\right]$ for $L_{4 r+2,4 x+2,4 t+1,3}$. Therefore $x_{0}=2 r+1, x_{1}=2 r+1$, $a_{0}=2 r, a_{1}=2 r+2, y_{0}=2 x, y_{1}=2 x+1, b_{0}=2 x+2$, $b_{1}=2 x, z_{0}=2 t, z_{1}=2 t, c_{0}=2 t+1, c_{1}=2 t, h_{0}=1$, $h_{1}=1, d_{0}=1$ and $d_{1}=2$. Hence $v_{0}-v_{1}=\left(x_{0}-x_{1}\right)+$ $\left(y_{0}-y_{1}\right)+\left(z_{0}-z_{1}\right)+\left(h_{0}-h_{1}\right)=-1$ and $e_{0}-e_{1}=$ 
$\left(a_{0}-a_{1}\right)+\left(b_{0}-b_{1}\right)+\left(c_{0}-c_{1}\right)+\left(d_{0}-d_{1}\right)=0$. Thus $L_{4 r+2,4 x+2,4 t+1,3}$ is cordial. Now, we need to study the following subcases for $s \geq 4$.

Subcase (1.2.17): $s \equiv 0(\bmod 4)$.

$L_{6,6,5,4}$ is cordial since it 's isomorphic to $L_{4,4,4,5}$.

Subcase $(2.2 .17): s \equiv 2(\bmod 4)$.

Suppose that $s=4 b+2$, where $b \geq 1$. We choose the labeling $\left[\bar{L}_{4 r} 10: L_{4 x} 1: L_{4 t}: S_{4 b} 0\right]$ for $L_{4 r+2,4 x+2,4 t+1,4 b+2}$. Therefore $x_{0}=2 r+1, x_{1}=2 r+1, a_{0}=2 r, a_{1}=2 r+$ $2, y_{0}=2 x, y_{1}=2 x+1, b_{0}=2 x+2, b_{1}=2 x, z_{0}=2 t$, $z_{1}=2 t, \quad c_{0}=2 t+1, \quad c_{1}=2 t, h_{0}=2 b+1, h_{1}=2 b$, $d_{0}=2 b$ and $d_{1}=2 b+2$. Hence $v_{0}-v_{1}=\left(x_{0}-x_{1}\right)+$ $\left(y_{0}-y_{1}\right)+\left(z_{0}-z_{1}\right)+\left(h_{0}-h_{1}\right)=0$ and $e_{0}-e_{1}=$ $\left(a_{0}-a_{1}\right)+\left(b_{0}-b_{1}\right)+\left(c_{0}-c_{1}\right)+\left(d_{0}-d_{1}\right)=-1$. Thus $L_{4 r+2,4 x+2,4 t+1,4 b+2}$ is cordial.

Subcase (3.2.17): $s \equiv 3(\bmod 4)$.

Suppose that $s=4 b+3$, where $b \geq 1$. We choose the labeling $\left[\bar{L}_{4 r} 10: L_{4 x} 1: L_{4 t}: 00 S_{4 b}\right]$ for $L_{4 r+2,4 x+2,4 t+1,4 b+3}$. Therefore $x_{0}=2 r+1, x_{1}=2 r+1, a_{0}=2 r, a_{1}=2 r+$ $2, y_{0}=2 x, y_{1}=2 x+1, b_{0}=2 x+2, b_{1}=2 x, z_{0}=2 t$, $z_{1}=2 t, \quad c_{0}=2 t+1, c_{1}=2 t, h_{0}=2 b+2, h_{1}=2 b$, $d_{0}=2 b+1$ and $d_{1}=2 b+2$. Hence $v_{0}-v_{1}=\left(x_{0}-\right.$ $\left.x_{1}\right)+\left(y_{0}-y_{1}\right)+\left(z_{0}-z_{1}\right)+\left(h_{0}-h_{1}\right)=1$ and $e_{0}-$ $e_{1}=\left(a_{0}-a_{1}\right)+\left(b_{0}-b_{1}\right)+\left(c_{0}-c_{1}\right)+\left(d_{0}-d_{1}\right)=0$

Thus $L_{4 r+2,4 x+2,4 t+1,4 b+3}$ is cordial.

Case (3.17): $k \equiv 2(\bmod 4)$.

We choose the labeling $\left[L_{4 r} 10: \bar{L}_{4 x} 1: L_{4 t} 0: 10\right]$ for $L_{4 r+2,4 x+2,4 t+2,3}$. Therefore $x_{0}=2 r+1, x_{1}=2 r+1$, $a_{0}=2 r+2, a_{1}=2 r, y_{0}=2 x, y_{1}=2 x+1, b_{0}=2 x$, $b_{1}=2 x+2, z_{0}=2 t+1, z_{1}=2 t, c_{0}=2 t+2, c_{1}=2 t$, $h_{0}=1, h_{1}=1, d_{0}=1$ and $d_{1}=2$. Hence $v_{0}-v_{1}=$ $\left(x_{0}-x_{1}\right)+\left(y_{0}-y_{1}\right)+\left(z_{0}-z_{1}\right)+\left(h_{0}-h_{1}\right)=0 \quad$ and $e_{0}-e_{1}=\left(a_{0}-a_{1}\right)+\left(b_{0}-b_{1}\right)+\left(c_{0}-c_{1}\right)+\left(d_{0}-d_{1}\right)=$ 1 . Thus $L_{4 r+2,4 x+2,4 t+2,3}$ is cordial. Now, we need to study the following subcases for $s>4$.

Subcase (1.3.17): $s \equiv 1(\bmod 4)$.

$L_{6,6,6,5}$ is cordial since it's isomorphic to $L_{6,6,5,6}$.

Subcase (2.3.17): $s \equiv 2(\bmod 4)$.

$L_{6,6,6,6}$ is cordial since it's the same as $L_{6,6,6,6}$.

Subcase $(3.3 .17): s \equiv 3(\bmod 4)$.

Suppose that $s=4 b+3$, where $b \geq 1$. We choose the labeling $\left[L_{4 r} 10: \bar{L}_{4 x} 1: L_{4 t} 0: 10 \bar{L}_{4 b}\right]$ for $L_{4 r+2,4 x+2,4 t+2,4 b+3}$. Therefore $x_{0}=2 r+1, \quad x_{1}=2 r+1, \quad a_{0}=2 r+2$, $a_{1}=2 r, y_{0}=2 x, y_{1}=2 x+1, b_{0}=2 x, b_{1}=2 x+2$, $z_{0}=2 t+1, z_{1}=2 t, c_{0}=2 t+2, c_{1}=2 t, h_{0}=2 b+1$, $h_{1}=2 b+1, d_{0}=2 b+1$ and $d_{1}=2 b+2$. Hence $v_{0}-v_{1}=\left(x_{0}-x_{1}\right)+\left(y_{0}-y_{1}\right)+\left(z_{0}-z_{1}\right)+\left(h_{0}-\right.$ $\left.h_{1}\right)=0$ and $e_{0}-e_{1}=\left(a_{0}-a_{1}\right)+\left(b_{0}-b_{1}\right)+\left(c_{0}-\right.$ $\left.c_{1}\right)+\left(d_{0}-d_{1}\right)=1$. Thus $L_{4 r+2,4 x+2,4 t+2,4 b+3}$ is cordial.

Case (4.17): $k \equiv 3(\bmod 4)$.

$L_{6,6,7,3}$ is not cordial. Now, we need to study the following subcases for $s \geq 4$.

Subcase (1.4.17): $s \equiv 0(\bmod 4)$.

$L_{6,6,7,4}$ is cordial since it ' $S$ isomorphic to $L_{6,6,4,7}$.

Subcase (2.4.17): $s \equiv 1(\bmod 4)$.

$L_{6,6,7,5}$ is cordial since it 's isomorphic to $L_{6,6,5,7}$.

Subcase $(3.4 .17): s \equiv 2(\bmod 4)$.
$L_{6,6,7,6}$ is cordial since it 's isomorphic to $L_{6,6,6,7}$.

Lemma 3.18 . If $n, m \equiv 2(\bmod 4)$ and $k \equiv i(\bmod 4)$, where $i=0,1,2,3$ then the four-leaved rose graph is not cordial if $(k+s \equiv 2(\bmod 4))$ for all $s \geq 3$.

Proof. It's easy to verify that the degree of all vertices of $L_{n, m, k, s}$ is even, and $L_{n, m, k, s}$ is an Eulerian graph with size is congruent to $2(\bmod 4)$, then from Cahit's theorem [7], $L_{n, m, k, s}$ is not cordial.

Lemma 3.19 . If $n, m \equiv 3(\bmod 4)$ and $k \equiv i(\bmod 4)$, where $i=0,1,2,3$ then the four-leaved rose graph is cordial except if $(k+s \equiv 0(\bmod 4))$ for all $s \geq 3$.

Proof. Suppose that $s \equiv\{3, j(\bmod 4)\}$, where $j=0,1,2,3$. We need to study the following cases:

Case (1.19): $k \equiv 0(\bmod 4)$.

We choose the labeling [ $\left.L_{4 r} 011: 0 L_{4 x} 1: L_{4 t} 100: 10\right]$ for $L_{4 r+3,4 x+3,4 t, 3}$. Therefore $x_{0}=2 r+1, x_{1}=2 r+2$, $a_{0}=2 r+1, a_{1}=2 r+2, y_{0}=2 x+1, y_{1}=2 x+1$, $b_{0}=2 x+3, b_{1}=2 x, z_{0}=2 t+2, z_{1}=2 t+1, c_{0}=$ $2 t+2, c_{1}=2 t+2, h_{0}=1, h_{1}=1, d_{0}=1$ and $d_{1}=2$. Hence $\quad v_{0}-v_{1}=\left(x_{0}-x_{1}\right)+\left(y_{0}-y_{1}\right)+\left(z_{0}-z_{1}\right)+$ $\left(h_{0}-h_{1}\right)=0$ and $e_{0}-e_{1}=\left(a_{0}-a_{1}\right)+\left(b_{0}-b_{1}\right)+$ $\left(c_{0}-c_{1}\right)+\left(d_{0}-d_{1}\right)=1$. Thus $L_{4 r+3,4 x+3,4 t, 3}$ is cordial.

Now, we need to study the following subcases for $s>4$.

Subcase (1.1.19): $s \equiv 1(\bmod 4)$.

Suppose that $s=4 b+1$, where $b \geq 1$. We choose the labeling [ $L_{4 r} 011: 0 L_{4 x} 1: L_{4 t} 100: \bar{S}_{4 b}$ ] for $L_{4 r+3,4 x+3,4 t, 4 b+1}$. Therefore $x_{0}=2 r+1, \quad x_{1}=2 r+2, \quad a_{0}=2 r+1$, $a_{1}=2 r+2, y_{0}=2 x+1, y_{1}=2 x+1, b_{0}=2 x+3$, $b_{1}=2 x, z_{0}=2 t+2, z_{1}=2 t+1, c_{0}=2 t+2, c_{1}=$ $2 t+2, h_{0}=2 b, h_{1}=2 b, d_{0}=2 b-1$ and $d_{1}=2 b+2$. Hence $\quad v_{0}-v_{1}=\left(x_{0}-x_{1}\right)+\left(y_{0}-y_{1}\right)+\left(z_{0}-z_{1}\right)+$ $\left(h_{0}-h_{1}\right)=0$ and $e_{0}-e_{1}=\left(a_{0}-a_{1}\right)+\left(b_{0}-b_{1}\right)+$ $\left(c_{0}-c_{1}\right)+\left(d_{0}-d_{1}\right)=-1$. Thus $L_{4 r+3,4 x+3,4 t, 4 b+1}$ is cordial.

Subcase (2.1.19): $s \equiv 2(\bmod 4)$.

Suppose that $s=4 b+2$, where $b \geq 1$. We choose the labeling $\quad\left[L_{4 r} 011: 0 L_{4 x} 1: L_{4 t} 100: 0 \bar{S}_{4 b}\right]$ for $L_{4 r+3,4 x+3,4 t, 4 b+2}$. Therefore $x_{0}=2 r+1, x_{1}=2 r+2$, $a_{0}=2 r+1, a_{1}=2 r+2, y_{0}=2 x+1, y_{1}=2 x+1$, $b_{0}=2 x+3, b_{1}=2 x, z_{0}=2 t+2, z_{1}=2 t+1, c_{0}=$ $2 t+2, c_{1}=2 t+2, h_{0}=2 b+1, h_{1}=2 b, d_{0}=2 b$ and $d_{1}=2 b+2$. Hence $v_{0}-v_{1}=\left(x_{0}-x_{1}\right)+\left(y_{0}-y_{1}\right)+$ $\left(z_{0}-z_{1}\right)+\left(h_{0}-h_{1}\right)=1$ and $e_{0}-e_{1}=\left(a_{0}-a_{1}\right)+$ $\left(b_{0}-b_{1}\right)+\left(c_{0}-c_{1}\right)+\left(d_{0}-d_{1}\right)=0$

$L_{4 r+3,4 x+3,4 t, 4 b+2}$ is cordial.

Subcase (3.1.19): $s \equiv 3(\bmod 4)$.

Suppose that $s=4 b+3$, where $b \geq 1$. We choose the labeling $\quad\left[L_{4 r} 011: 0 L_{4 x} 1: 001 L_{4 t}: 01 L_{4 b}\right]$ for $L_{4 r+3,4 x+3,4 t, 4 b+3}$. Therefore $x_{0}=2 r+1, x_{1}=2 r+2$, $a_{0}=2 r+1, a_{1}=2 r+2, y_{0}=2 x+1, y_{1}=2 x+1$, $b_{0}=2 x+3, b_{1}=2 x, z_{0}=2 t+2, z_{1}=2 t+1, c_{0}=$ $2 t+2, \quad c_{1}=2 t+2, h_{0}=2 b+1, h_{1}=2 b+1, d_{0}=$ $2 b+1$ and $d_{1}=2 b+2$. Hence $v_{0}-v_{1}=\left(x_{0}-x_{1}\right)+$ $\left(y_{0}-y_{1}\right)+\left(z_{0}-z_{1}\right)+\left(h_{0}-h_{1}\right)=0$ and $e_{0}-e_{1}=$ $\left(a_{0}-a_{1}\right)+\left(b_{0}-b_{1}\right)+\left(c_{0}-c_{1}\right)+\left(d_{0}-d_{1}\right)=1$. Thus $L_{4 r+3,4 x+3,4 t, 4 b+3}$ is cordial.

Case $(2.19): k \equiv 1(\bmod 4)$. 
$L_{7,7,5,4}$ is not cordial. Now, we need to study the following subcases for $s \geq 4$.

Subcase (1.2.19): $s \equiv 0(\bmod 4)$.

$L_{7,7,5,4}$ is cordial since it ' $S$ isomorphic to $L_{7,7,4,5}$.

Subcase (2.2.19): $s \equiv 1(\bmod 4)$.

$L_{7,7,5,5}$ is cordial since it ' $S$ isomorphic to $L_{5,5,7,7}$.

Thus $L_{4 r+2,4 x+2,4 t+1,4 b+2}$ is cordial.

Subcase (3.2.19): $s \equiv 2(\bmod 4)$.

Suppose that $s=4 b+2$, where $b \geq 1$. We choose the labeling [ $\left.L_{4 r} 011: 0 L_{4 x} 1: L_{4 t}: 0 \bar{S}_{4 b}\right]$ for $L_{4 r+3,4 x+3,4 t+1,4 b+2}$. Therefore $x_{0}=2 r+1, \quad x_{1}=2 r+2, \quad a_{0}=2 r+1$, $a_{1}=2 r+2, y_{0}=2 x+1, y_{1}=2 x+1, b_{0}=2 x+3$, $b_{1}=2 x, z_{0}=2 t, z_{1}=2 t, c_{0}=2 t+1, c_{1}=2 t, h_{0}=$ $2 b+1, h_{1}=2 b, d_{0}=2 b$ and $d_{1}=2 b+2$. Hence $v_{0}-v_{1}=\left(x_{0}-x_{1}\right)+\left(y_{0}-y_{1}\right)+\left(z_{0}-z_{1}\right)+\left(h_{0}-\right.$ $\left.h_{1}\right)=0$ and $e_{0}-e_{1}=\left(a_{0}-a_{1}\right)+\left(b_{0}-b_{1}\right)+\left(c_{0}-\right.$ $\left.c_{1}\right)+\left(d_{0}-d_{1}\right)=1$. Thus $L_{4 r+3,4 x+3,4 t+1,4 b+2}$ is cordial.

Case (3.19): $k \equiv 2(\bmod 4)$.

We choose the labeling $\left[L_{4 r} 011: 0 L_{4 x} 1: L_{4 t} 0: 10\right]$ for $L_{4 r+3,4 x+3,4 t+2,3}$. Therefore $x_{0}=2 r+1, x_{1}=2 r+2$, $a_{0}=2 r+1, a_{1}=2 r+2, y_{0}=2 x+1, y_{1}=2 x+1$, $b_{0}=2 x+3, b_{1}=2 x, z_{0}=2 t+1, z_{1}=2 t, c_{0}=2 t$, $c_{1}=2 t+2, h_{0}=1, h_{1}=1, d_{0}=1$ and $d_{1}=2$. Hence $v_{0}-v_{1}=\left(x_{0}-x_{1}\right)+\left(y_{0}-y_{1}\right)+\left(z_{0}-z_{1}\right)+\left(h_{0}-\right.$ $\left.h_{1}\right)=0$ and $e_{0}-e_{1}=\left(a_{0}-a_{1}\right)+\left(b_{0}-b_{1}\right)+\left(c_{0}-\right.$ $\left.c_{1}\right)+\left(d_{0}-d_{1}\right)=-1$. Thus $L_{4 r+3,4 x+3,4 t+2,3}$ is cordial.

Now, we need to study the following subcases for $s \geq 4$.

Subcase (1.3.19): $s \equiv 0(\bmod 4)$.

$L_{7,7,6,4}$ is cordial since it ' $\mathrm{S}$ isomorphic to $L_{7,7,4,6}$.

Subcase (2.3.19): $s \equiv 1(\bmod 4)$.

$L_{7,7,6,5}$ is cordial since it's isomorphic to $L_{7,7,5,6}$.

Subcase (3.3.19): $s \equiv 3(\bmod 4)$.

Suppose that $s=4 b+3$, where $b \geq 1$. We choose the labeling $\quad\left[L_{4 r} 011: 0 \bar{L}_{4 x} 1: 0 L_{4 t}: 1 L_{4 b} 0\right] \quad$ for $L_{4 r+3,4 x+3,4 t+2,4 b+3}$. Therefore $x_{0}=2 r+1, x_{1}=2 r+1$, $a_{0}=2 r+1, a_{1}=2 r+2, y_{0}=2 x+1, y_{1}=2 x+1$, $b_{0}=2 x+1, b_{1}=2 x+2, z_{0}=2 t+1, z_{1}=2 t, c_{0}=$ $2 t+2, c_{1}=2 t, h_{0}=2 b+1, h_{1}=2 b+1, d_{0}=2 b+1$ and $d_{1}=2 b+2$. Hence $v_{0}-v_{1}=\left(x_{0}-x_{1}\right)+\left(y_{0}-\right.$ $\left.y_{1}\right)+\left(z_{0}-z_{1}\right)+\left(h_{0}-h_{1}\right)=0$ and $e_{0}-e_{1}=\left(a_{0}-\right.$ $\left.a_{1}\right)+\left(b_{0}-b_{1}\right)+\left(c_{0}-c_{1}\right)+\left(d_{0}-d_{1}\right)=-1$. Thus

$L_{4 r+3,4 x+3,4 t+2,4 b+3}$ is cordial.

Case (4.19): $k \equiv 3(\bmod 4)$.

We choose the labeling $\left[L_{4 r} 101: 0 \bar{L}_{4 x} 1: 10 L_{4 t}: 10\right]$ for $L_{4 r+3,4 x+3,4 t+3,3}$. Therefore $x_{0}=2 r+1, x_{1}=2 r+2$, $a_{0}=2 r+1, a_{1}=2 r+2, y_{0}=2 x+1, y_{1}=2 x+1$, $b_{0}=2 x+1, \quad b_{1}=2 x+2, \quad z_{0}=2 t+1, z_{1}=2 t+1$, $c_{0}=2 t+3, c_{1}=2 t, h_{0}=1, h_{1}=1, d_{0}=1$ and $d_{1}=2$. Hence $\quad v_{0}-v_{1}=\left(x_{0}-x_{1}\right)+\left(y_{0}-y_{1}\right)+\left(z_{0}-z_{1}\right)+$ $\left(h_{0}-h_{1}\right)=-1$ and $e_{0}-e_{1}=\left(a_{0}-a_{1}\right)+\left(b_{0}-b_{1}\right)+$ $\left(c_{0}-c_{1}\right)+\left(d_{0}-d_{1}\right)=0$. Thus $L_{4 r+3,4 x+3,4 t+3,3}$ is cordial.

Now, we need to study the following subcases for $s \geq 4$.

Subcase (1.4.19): $s \equiv 0(\bmod 4)$.

$L_{7,7,7,4}$ is cordial since it ' $\mathrm{s}$ isomorphic to $L_{7,7,4,7}$.

Subcase (2.4.19): $s \equiv 2(\bmod 4)$.

$L_{7,7,7,6}$ is cordial since it 's isomorphic to $L_{7,7,6,7}$.

Subcase (3.4.19): $s \equiv 3(\bmod 4)$.
$L_{7,7,7,7}$ is cordial since it 's isomorphic to $L_{7,7,7,7}$.

Lemma 3.20 . If $n, m \equiv 3(\bmod 4)$ and $k \equiv i(\bmod 4)$, where $i=0,1,2,3$ then the four-leaved rose graph is not cordial if $(k+s \equiv 0(\bmod 4))$ for all $s \geq 3$.

Proof. It's easy to verify that the degree of all vertices of $L_{n, m, k, s}$ is even, and $L_{n, m, k, s}$ is an Eulerian graph with size is congruent to $2(\bmod 4)$, then from Cahit's theorem [7], $L_{n, m, k, s}$ is not cordial.

As a consequence of all previous lemmas one can establish the following theorem.

Theorem 3.1. The four-leaved rose graph is cordial for all $n, m, k$ and $s$ except if $n, m$ are odd and $(k+s) \equiv$ $0(\bmod 4)$ or $n, m$ are even and $(k+s) \equiv 2(\bmod 4)$.

In the four-leaved rose graph $L_{n, m, k, s}$, the number of vertices is $|V|=n+m+k+s-3$, and the number of edges is $|E|=n+m+k+s$. The following conclusion are consequence of the previous lemmas:

$L_{n, m, k}$ is cordial in 2 cases:

1. If $n, m, k$ and $s$ are odd or even, then $|V|$ is odd, $V_{0}, V_{1}=\left(\frac{n+m+k+s-4}{2}\right)$ or $\frac{n+m+k+s-2}{2},|E|$ is even and $E_{0}=E_{1}=\frac{n+m+k+s}{2}$.

2. If $n, m, k$ and $s$ are different, then $|V|$ is even, $V_{0}, V_{1}=\left(\frac{n+m+k+s-3}{2}\right),|E|$ is odd and $E_{0}, E_{1}=\frac{n+m+k+s+1}{2}$ or $\frac{n+m+k+s-1}{2}$.

\section{Conclusion}

We show that any four-leaved rose graph is cordial for all $n, m, k$ and $s$ except possibly at $n, m$ are odd and $(k+s) \equiv$ $0(\bmod 4)$ or $n, m$ are even and $(k+s) \equiv 2(\bmod 4)$.

\section{References}

[1] Rosa, A. , On certain valuations of the vertices of a graph, Theory of Graphs( Internat Symposium, Rome, July 1966),Gordon and Breach, N.Y.and Dunod Paris, (1967) 349- 355.

[2] Graham, R. L. and Sloane, N.J.A., On additive bases and harmonious graphs, SIAM J. Alg. Discrete Math. 1, (1980) 382-404.

[3] Elrokh, A. and Atef Mohamed, The cordiality of lemniscate graph and its second power, Malaysian journal of mathematical science, 2017 under review

[4] Elrokh, A., The cordiality of the three-leaved rose graph, submitted 2018.

[5] Diab, A. T., On Cordial Labelings of Wheels with Other Graphs, ARS Combinatoria 100, (2011) 265-279.

[6] Golomb, S. W., How to number a graph in Graph Theory and Computing,R.C. Read, ed., Academic Press, New York, (1972) 23-37.

[7] Gallian, J. A. , A dynamic survey of graph labeling, The Electronic Journal of Combina- torics 17, December 29 (2014).

[8] Cahit, I. , Cordial Graphs: A Weaker Version of Graceful and Harmonious Graphs, Ars Combin. 23(1987) 201-207. 
[9] Kirchherr, W. W., On the cordiality of some specific graphs, ARS Combinatoria 31(1991), pp 127-138.
[10] Lee, S. M. and Liu, A. , A construction of cordial graphs from smaller cordial graphs, Ars Combin., 32(1991) 209-214. 